\title{
Artesunate induces apoptosis and autophagy in HCT116 colon cancer cells, and autophagy inhibition enhances the artesunate-induced apoptosis
}

\author{
FENG JIANG $^{1,2}$, JIN-YONG ZHOU ${ }^{3}$, DAN ZHANG $^{1,2}$, MING-HAO LIU $^{2}$ and YU-GEN CHEN ${ }^{1}$ \\ ${ }^{1}$ Department of Colorectal Surgery, The Affiliated Hospital of Nanjing University of TCM, Nanjing, Jiangsu 210029; \\ ${ }^{2}$ No. 1 Clinical Medical College, Nanjing University of Chinese Medicine, Nanjing, Jiangsu 210023; \\ ${ }^{3}$ Department of Central Laboratory, The Affiliated Hospital of Nanjing University of TCM, \\ Nanjing, Jiangsu 210029, P.R. China
}

Received December 18,2017; Accepted May 30, 2018

DOI: $10.3892 / \mathrm{ijmm} .2018 .3712$

\begin{abstract}
The present study assessed the antitumor effect of artesunate (ART) in vitro and in vivo, as well as its underlying mechanism of action in HCT116 colon cancer cells. An MTT assay, DAPI staining, flow cytometry, western blotting, immunohistochemistry, transmission electron microscopy and TUNEL assay were performed to study the molecular mechanism underlying the antitumor effects of ART in HCT116 colon cancer cells. ART was observed to inhibit proliferation by inducing the apoptosis of HCT116 cells both in vitro and in vivo. Flow cytometry analysis demonstrated that treatment with 2 and $4 \mu \mathrm{g} / \mathrm{ml}$ ART for $48 \mathrm{~h}$ induced early apoptosis in 22.7 and $33.8 \%$ of cells, respectively. In the xenograft tumors of BALB/c nude mice, TUNEL-positive cells increased in the ART group compared with that in the normal saline group. Furthermore,the associated mitochondrial cleaved-caspase 3, poly-ADP ribose polymerase (PARP), caspase 9 and $\mathrm{Bcl}-2$-associated $\mathrm{X}$ protein levels increased while B-cell lymphoma-2 (Bcl-2) decreased both in the cell and animal ART-treated group. ART-treated cells also exhibited autophagy induction, as evidenced by increased protein expression levels of light chain 3 (LC3) and beclin-1, and the presence of autophagosomes. Notably, pharmacological blockade of autophagy activation using hydroxychloroquine markedly enhanced ART-induced apoptosis and increased the
\end{abstract}

Correspondence to: Dr Yu-Gen Chen, Department of Colorectal Surgery, The Affiliated Hospital of Nanjing University of TCM, 155 Hanzhong Road, Nanjing, Jiangsu 210029, P.R. China

E-mail: chenyg666@126.com

Abbreviations: ART, artesunate; Bcl-2, B-cell lymphoma-2; Bax, $\mathrm{Bcl}-2$ associated $\mathrm{X}$ protein; Bcl-xL, Bcl-extra-large protein; CRC, colorectal cancer; DAPI, 4',6-diamidino-2-phenylindole; HCQ, hydroxychloroquine sulfate; TUNEL, TdT-mediated dUTP nick end labelling; MTT, 3-(4,5-dimethylthiazol-2-yl)2,5-diphenyl tetrazolium bromide; PARP, poly-ADP ribose polymerase

Key words: apoptosis, artesunate, autophagy, colon cancer protein levels of cleaved caspase 3 and PARP, while decreasing the levels of LC3 and beclin-1. These findings suggested that the ART-induced autophagy may have a cytoprotective effect by suppressing apoptosis. In conclusion, ART may be a potentially clinically useful anticancer drug for human colon cancer. In addition, co-treatment with ART and an autophagy inhibitor may be an effective anticancer therapy.

\section{Introduction}

Colorectal cancer (CRC) is considered as one of the most common malignancies with a high incidence and mortality worldwide. It is estimated that 1.4 million individuals were newly diagnosed with CRC in 2012, which resulted in 693,900 mortalities (1). Surgery, chemotherapy and radiation treatment are the three main standard therapies for CRC. Chemotherapy is an important treatment for cancer, particularly in tumors with a propensity to invade adjacent tissues and metastasize to other organs. The introduction of advanced surgical and systemic therapeutic options has improved the prognosis for CRC over the past few years. However, challenges remain that require the continued search for novel effective and less toxic chemotherapeutic agents for the treatment of colon cancer (2).

Artesunate (ART), a natural sesquiterpene extracted from a Chinese medicinal herb, is recognized as a safe compound for treating malaria (3). It is considered to be suitable for drug development due to its aqueous solubility. In 1996, Efferth et al (4) discovered for the first time that ART was able to induce tumor cell apoptosis. Currently, the potential anticancer effects of ART have been reported in numerous tumors, including myelodysplastic syndrome $(5,6)$, multiple myeloma (7), chronic myeloid leukemia (8), Burkitt's lymphoma (9), bladder cancer (10), renal carcinoma (11), cervical (12), breast (13), prostate (14), and head and neck (15) cancer, hepatocellular carcinoma (16), esophageal cancer (17) and CRC (18). However, the specific mechanism of its antitumor activity remain unclear.

Presently, the ART-induced tumor cell death has been reported to mainly involve apoptosis $(5,16,18,19)$, 
autophagy (13), oncosis (11) and ferroptosis (15). Cell death signaling is complex, and the different processes function in concert. Apoptosis and autophagy are two distinct processes that coordinately regulate cell survival and death, and occur simultaneously in numerous types of cancer (20). Furthermore, these two processes have been identified in CRC (21).

It has been reported that ART induces apoptosis and autophagic excitation of Raji cells in Burkitt's lymphoma (9) and suppresses HCT116 cell proliferation through apoptosis pathways (18). Therefore, in the present study, the effects of ART on apoptosis and autophagy of HCT116 cells were investigated, and the association between apoptosis and autophagy was explored in vitro. In addition, the inhibitory effect of ART on subcutaneous xenografts was assessed in nude mice.

\section{Materials and methods}

Cells and animals. The human HCT116 CRC cell line was routinely cultured at the Centre Laboratory of the Affiliated Hospital of Nanjing University of TCM. The cells were cultured in RPMI 1640 medium (HyClone; Thermo Fisher Scientific, Inc., Waltham, MA, USA) supplemented with $10 \%$ fetal calf serum (Evergreen; Zhejiang Tianhang Biotechnology Co., Ltd, Luoshe, China). The cells were incubated at $37^{\circ} \mathrm{C}$ in a humidified atmosphere of $5 \% \mathrm{CO}_{2}$.

The experimental animal protocols were reviewed and approved by the Animal Ethics Committee of the Affiliated Hospital of Nanjing University of TCM (2017-DW-11). A total of 20 6-week-old BALB/c nude mice weighing 18-22 g (no. 201716339) were purchased from Changzhou Cavens Laboratory Animal Co., Ltd (Changzhou, China). They were maintained under specific pathogen-free conditions at room temperature $\left(23 \pm 2^{\circ} \mathrm{C}\right)$ and relative humidity $(40-60 \%)$, with a12 $\mathrm{h}$ light/dark cycle, and their cages, litter, food and water were strictly sterilized.

Materials. ART was purchased from Guilin Pharmaceutical Co., Ltd. (Guilin, China). According to the manufacturer's protocol, ART was dissolved to a concentration of $20 \mathrm{mg} / \mathrm{ml}$ and then stored at $-20^{\circ} \mathrm{C}$. Prior to the experiments, the ART stock was diluted to the final desired concentrations with complete RPMI 1640. 5-Fu was purchased from Tianjin King York Pharmaceutical Co., Ltd. (Tianjin, China).

Antibodies against caspase 3 (cat. no. 9665), caspase 9 (cat.no.9502),poly-ADPribose polymerase(PARP; cat.no.9532), B-cell lymphoma-2 (Bcl-2; cat. no. 2876), Bcl-extra-large (Bcl-xL; cat. no. 2764), Bcl-2-associated X protein (Bax; cat. no. 2772), light chain 3 (LC3)-I/II (cat. no. 12741), beclin-1 (cat. no. 3495), autophagy protein 5 (Atg5; cat. no. 12994), Atg12 (cat. no. 4180), $\beta$-actin (cat. no. 4970) and anti-rabbit IgG HRP-linked Antibody (cat. no. 7074P2) were purchased from Cell Signaling Technology, Inc., (Danvers, MA, USA). Primary antibody was purchased from Wuhan Servicebio Technology Co., Ltd. (Wuhan, China; cat. no. G2025), and the dilution ratio used for each antibody was 1:1,000. Hydroxychloroquine sulfate (HCQ) was purchased from Selleck Chemicals (Houston, TX, USA).

3-(4,5-Dimethylthiazol-2-yl)2,5-diphenyl tetrazolium bromide (MTT) assay. Cell viability was evaluated using an MTT assay. Briefly, the cells were seeded in 96-well plates at a cell density of 4,000 cells/well. Following treatment of the cells with ART at various concentrations (2.5, 10,40 and $80 \mu \mathrm{g} / \mathrm{ml}$ ) or the control for the indicated time (24, 48 and $72 \mathrm{~h}$ ), the MTT reagent was added to each well at a final concentration of $5 \mathrm{mg} / \mathrm{ml}$, followed by incubation at $37^{\circ} \mathrm{C}$ for $4 \mathrm{~h}$. Next, the medium was removed, and $150 \mu \mathrm{l}$ dimethyl sulfoxide was added to each well. The absorbance was measured at $490 \mathrm{~nm}$ using a microplate reader (BioTek Instruments, Inc., Winooski, VT, USA). The relative cell viability was calculated as follows: Relative cell viability $(\%)=($ Mean absorbance of the test wells/mean absorbance of the control wells) $x 100$. The half maximal inhibitory concentration $\left(\mathrm{IC}_{50}\right)$ was calculated using SPSS Software based on the results of MTT. Ranjan et al (22) identified the $\mathrm{IC}_{50}$ of penfluridol was $6.5 \mu \mathrm{M}$ in Panc-1 cells following $24 \mathrm{~h}$ treatment. Thus, $5 \mu \mathrm{M}$ and $10 \mu \mathrm{M}$ around $6.5 \mu \mathrm{M}$ were selected for the study. Wang et al (23) selected $20 \mu \mathrm{M}$ and $40 \mu \mathrm{M}$ emodin for the experiment in accordance with the results of $\mathrm{IC}_{50}$. In the present experiment, the $\mathrm{IC}_{50}$ of ART on HCT116 was $2.18 \mu \mathrm{g} / \mathrm{ml}$ following $48 \mathrm{~h}$. When the concentration of ART was $<1 \mu \mathrm{g} / \mathrm{ml}$, the inhibition was $<30 \%$, so $2 \mu \mathrm{g} / \mathrm{ml}$ was selected as the low dosage group, indicating $30-50 \%$ inhibition. Twice the low dosage was used for the high dosage group $(4 \mu \mathrm{g} / \mathrm{ml}$ ART), which resulted in $>50 \%$ inhibition.

Cell morphological changes examined using 4',6-diamidino2-phenylindole (DAPI) staining. HCT116 cells were treated with ART ( 2 and $4 \mu \mathrm{g} / \mathrm{ml})$ and $5-\mathrm{Fu}(0.3 \mu \mathrm{g} / \mathrm{ml})$ for $48 \mathrm{~h}$ respectively, fixed with $4 \%$ paraformaldehyde for $10 \mathrm{~min}$ at room temperature and then stained with DAPI (Beyotime Institute of Biotechnology, Shanghai, China) for $15 \mathrm{~min}$. Subsequently, the cells were observed under an LSM710 microscope (Carl Zeiss, Oberkochen, Germany).

Detection of apoptosis using flow cytometry. The apoptosis of treated cells was examined using an Annexin V-FITC/PI apoptosis kit (MultiSciences Biotech Co., Ltd., Hangzhou, China) according to the manufacturer's protocol. Briefly, the cells were collected and washed twice in phosphate-buffered saline (PBS). Cells were then labeled with $500 \mu \mathrm{l} 1 \mathrm{X}$ binding buffer containing $5 \mu \mathrm{l}$ Annexin V-fluorescein isothiocyanate (FITC) and $10 \mu \mathrm{l}$ propidium iodide (PI) in the dark for $5 \mathrm{~min}$. Subsequently, apoptosis was detected using a flow cytometer (BD Falcon; BD Biosciences, Franklin Lakes, NJ, USA), and the Annexin V and PI values were set as the horizontal and vertical axes, respectively, for the plot construction. Mechanically damaged, late apoptotic, dual negative/normal, and early apoptotic cells were located in the upper left, upper right, lower left and lower right quadrants of the flow cytometric dot plot, respectively.

Transmission electron microscopy (TEM) analysis. HCT116 cells were postfixed in $2.5 \%$ glutaraldehyde for $4 \mathrm{~h}$ at $4^{\circ} \mathrm{C}$ (Wuhan Goodbio Technology Co., Ltd, Wuhan, China), rinsed with PBS and then fixed in $1 \%$ osmium tetroxide $\left(\mathrm{OsO}_{4}\right.$; Sinopharm Chemical Reagents Co., Ltd., Shanghai, China) in $0.1 \mathrm{M}$ PBS for $1 \mathrm{~h}$ at room temperature. Following dehydration with ethanol (Sinopharm Chemical Reagent Co., Ltd., Shanghai, China), the cells were embedded in Epon resin (Sales 
Performance International Greater China Co., Beijing, China), and ultrathin sections of the selected areas were cut using the LKB NOVA ultramicrotome (Leica Biosystems, Solms, Germany) using a diamond knife (Daito Me Holdings Co., Ltd, Tokyo, Japan). Cells were then stained with a saturated solution of uranyl acetate in methanol (50:50) (Sinopharm Chemical Reagent Co., Ltd.) for $12 \mathrm{~min}$ at $45^{\circ} \mathrm{C}$, followed by incubation in an aqueous solution of concentrated bismuth subnitrate (Sinopharm Chemical Reagent Co., Ltd.) for $10 \mathrm{~min}$ at $25^{\circ} \mathrm{C}$. Subsequently, all the sections were examined under a Hitachi TEM system (Hitachi, Ltd., Tokyo, Japan).

Animal studies. HCT116 cells in the logarithmic growth phase were resuspended and digested to a density of $1 \times 10^{7}$ cells $/ \mathrm{ml}$. Each mouse was inoculated with $0.2 \mathrm{ml}$ of the cell suspension into the armpit. Approximately 2 weeks after inoculation, 15 mice that exhibited induration with a diameter of $8-10 \mathrm{~mm}$, which suggested that a successful tumor model was established, were randomly divided into three groups. Mice in the three groups were administered physiological saline [NS; $0.2 \mathrm{ml} /$ day, intraperitoneally (i.p.), every other day (qod)], 5-fluorouracil (5-Fu; $12.5 \mathrm{mg} / \mathrm{kg}$, i.p., qod) or ART (100 mg/kg, i.p., daily), respectively, for 2 weeks.

Observation of antitumor effects. The daily diet, defecation, urination, activity status and tumor growth of the mice were observed. Subsequent to treatment, the short- and long-axis diameters of the tumors were measured every other day, and the tumor volumes were calculated as follows using the Steel formula (17): Tumor volume $(\mathrm{V})\left(\mathrm{cm}^{3}\right)=($ long diameter $\mathrm{x}$ short diameter $\left.{ }^{2}\right) / 2$. The volume inhibition rate (\%) was also determined as follows: $\left(1-\mathrm{V}_{\text {experimental group }} / \mathrm{V}_{\text {control group }}\right) \times 100 \%$. The mice were euthanized on day 14 after drug administration. The primary tumors were excised, and the tumor volumes were measured as $V=4 / 3 \pi r^{3}$, where $r$ is the mean radius of three measurements. Half of the tumor tissue was fixed in formalin for $24 \mathrm{~h}$ at room temperature and embedded in paraffin for TdT-mediated dUTP nick end labelling (TUNEL) and immunohistochemical assays, while the other part of the tumor was snap frozen in liquid nitrogen and stored at $-80^{\circ} \mathrm{C}$ for western blotting analysis.

Immunohistochemistry. Paraffin-embedded tumor tissue samples were cut into $3-\mu \mathrm{m}$ sections, deparaffinized and subjected to antigen recovery with citric acid buffer (Wuhan Servicebio Technology Co., Ltd.) under high pressure for $2 \mathrm{~min}$. Following blocking with goat serum (OriGene Technologies, Inc., Beijing, China) at room temperature for $10 \mathrm{~min}$, then the samples were stained with rabbit anti-cleaved caspase 3 (1:300), anti-Bax (1:200) and anti-Bcl-2 (1:200) antibodies at $4^{\circ} \mathrm{C}$ overnight. 3,3'-Diaminobenzidine (Wuhan Servicebio Technology Co., Ltd.) was used to detect the immunocomplexes, whereas hematoxylin was used for nuclear counterstaining. An immunoglobulin-negative control was used to eliminate nonspecific binding. The sections were examined by light microscopy (Leica Biosystems, Solms, Germany) at x200 magnification. Images were analyzed using Image Pro Plus (version 6.0; Media Cybernetics, Inc., Rockville, MD, USA). The Integrated optical density from three fields of each slice was calculated using Intel IPP software (version 6.0; Intel, Mountain View,
CA, USA). Three fields were selected from the top to the bottom of each slice randomly.

TUNEL assay. Sections ( $3 \mu \mathrm{m}$ thick) cut from the paraffin-embedded tissue were dewaxed with xylene twice for 15 min, hydrated using an ethanol gradient (twice with $100 \%$ for $5 \mathrm{~min}$, then $85 \%$ for $5 \mathrm{~min}$ and $75 \%$ for $5 \mathrm{~min}$ ), fixed in $4 \%$ formaldehyde solution for $20 \mathrm{~min}$ at room temperature and then incubated with proteinase $\mathrm{K}$ at $37^{\circ} \mathrm{C}$ for $30 \mathrm{~min}$. The TUNEL assay kit (Roche Diagnostics, Basel, Switzerland) containing TdT was prepared immediately before use according to the manufacturer's protocol. Subsequent to washing with PBS, the sections were counterstained with DAPI. Apoptotic cells in the sections were detected using a microscope (Nikon Corp., Tokyo, Japan).

Western blot analysis. The cells and tumor tissues were lysed with ice-cold radio immunoprecipitation assay buffer containing a protease inhibitor and phosphatase inhibitors (KeyGen Biotech Co., Ltd., Nanjing, China). Protein concentrations were determined using a modified bovine serum albumin assay kit (Thermo Fisher Scientific, Inc.). Equal amounts of protein $(30 \mu \mathrm{g})$ from each sample were separated using $10 \%$ sodium dodecyl sulfate-polyacrylamide gel electrophoresis, followed by transfer onto polyvinylidene difluoride membranes (EMD Millipore, Billerica, MA, USA) and then incubation with 5\% BSA for $1 \mathrm{~h}$ at room temperature for blocking. The membranes were subsequently probed with different primary antibodies (Cell Signaling Technology, Inc.) at $4^{\circ} \mathrm{C}$ overnight. Next, the membranes were washed with Tris-buffered saline with Tween-20 (Sangon Biotech Co., Ltd. Shanghai, China) and incubated with anti-rabbit IgG HRP-conjugated antibody. An enhanced chemiluminescence kit (EMD Millipore) was subsequently used to detect the immunoreactive signals. Image J software (version 2.1; National Institutes of Health, Bethesda, MD, USA) was used analyze the results and calculate the expression of the protein.

Statistical analyses. The results are expressed as the mean \pm standard error of the mean. The data were analyzed using the t-test and one-way analysis of variance. Differences among the groups were analyzed using Duncan's multiple range test with the SPSS software (version 22.0; IBM Corp., Armonk, NY, USA). A value of $\mathrm{P}<0.05$ was considered to denote a statistically significant difference.

\section{Results}

ART inhibits the viability and growth of HCT116 cells. The structure of ART (Fig. 1A) was derived from the instructions of pharmaceutical company. Treatment of HCT116 cells with different concentrations of ART for various times inhibited the cell viability in a time- and concentration-dependent manner as determined by the MTT assay results (Table I). Upon treatment with different concentrations, the inhibition rates at 48 and $72 \mathrm{~h}$ were significantly different as compared with that at $24 \mathrm{~h}$ $(\mathrm{P}<0.01)$. Furthermore, at 48 and $72 \mathrm{~h}$, incubation with 40 and $80 \mu \mathrm{g} / \mathrm{ml}$ ART resulted in significantly inhibited cell viability when compared with that observed for the 2.5 and $10 \mu \mathrm{g} / \mathrm{ml}$ concentrations $(\mathrm{P}<0.05)$, whereas no significant difference was observed among the various concentrations at 24 h. (Fig. 1B). 
A

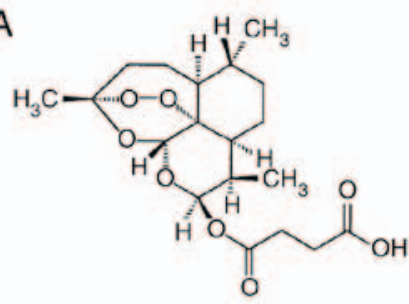

C

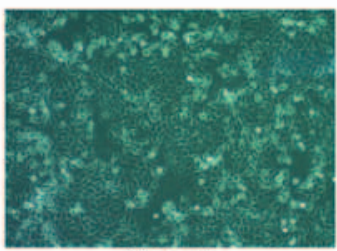

Control

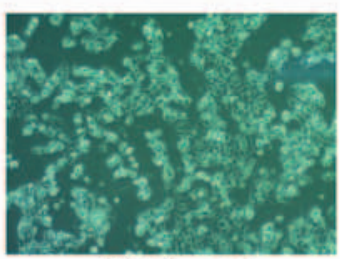

ART $(4 \mu \mathrm{g} / \mathrm{ml})$

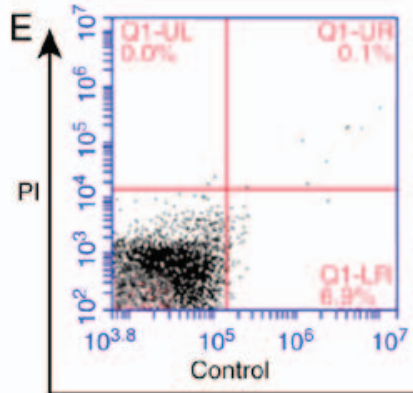

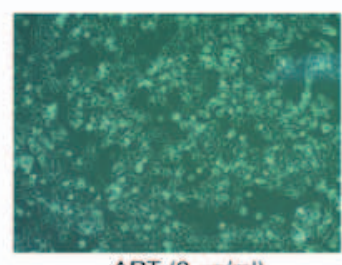

ART $(2 \mu \mathrm{g} / \mathrm{ml})$

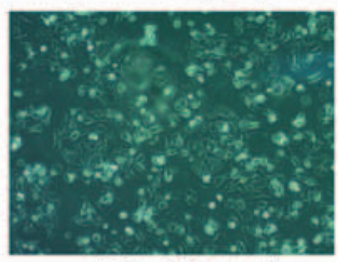

5-Fu $(0.3 \mu \mathrm{g} / \mathrm{ml})$

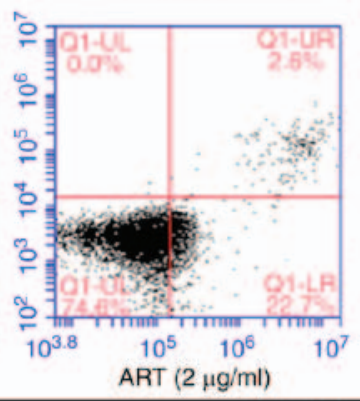

B
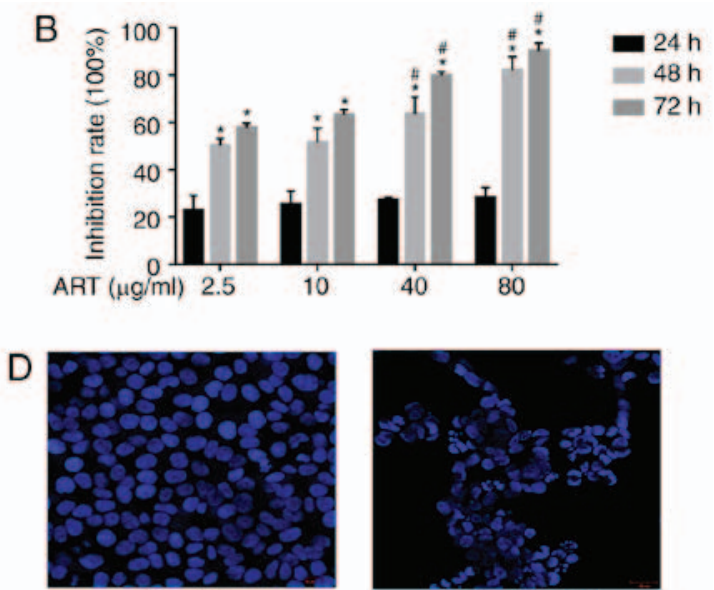

Control

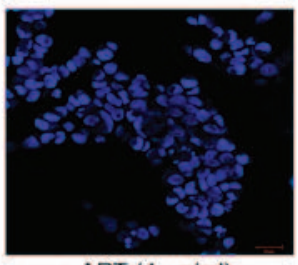

ART $(4 \mu \mathrm{g} / \mathrm{ml})$

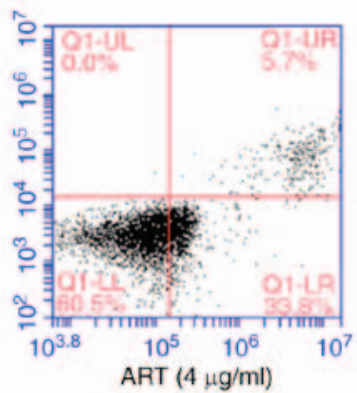

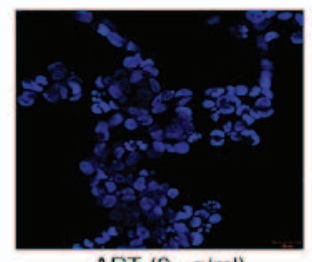

ART $(2 \mu \mathrm{g} / \mathrm{ml})$

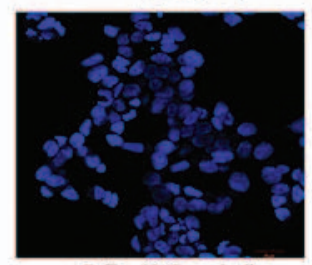

$5-\mathrm{Fu}(0.3 \mu \mathrm{g} / \mathrm{ml})$

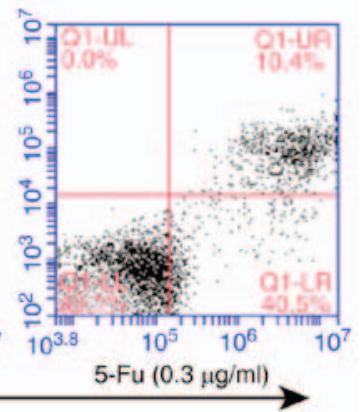

Annexin V
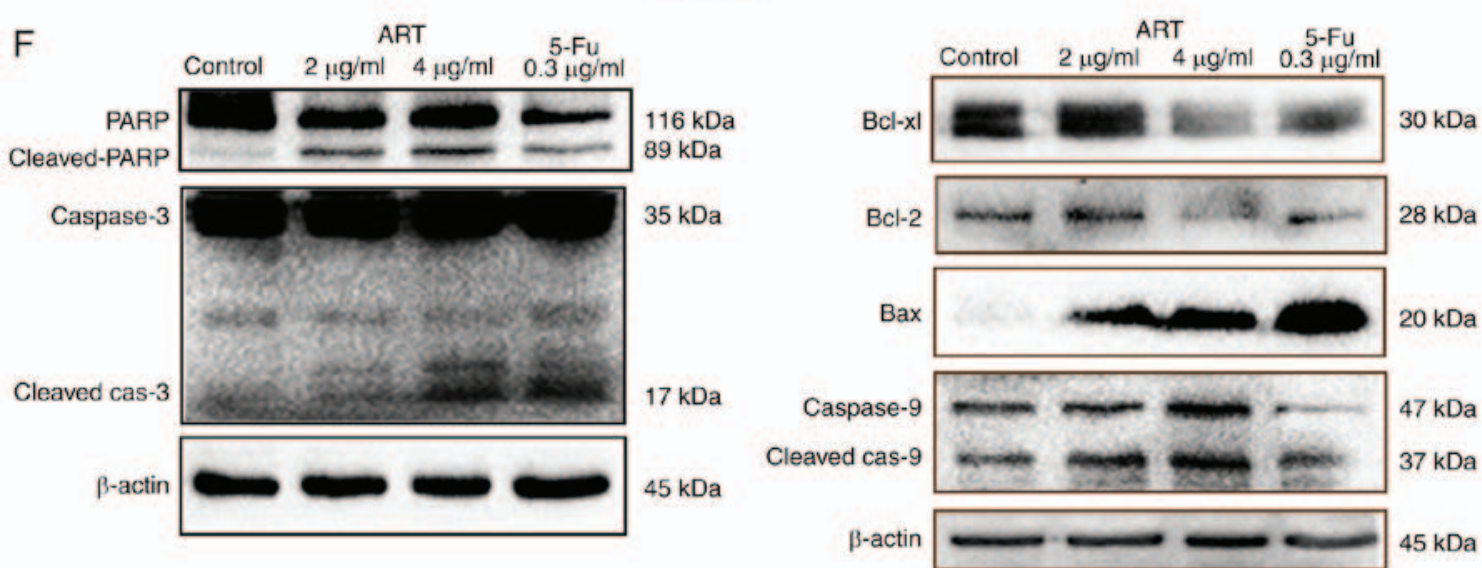

Figure 1. ART inhibits the growth of HCT116 cells and induces apoptosis. (A) Structure of ART. (B) Cell viability of HCT116 cells treated with ART (2.5, 10, 40 and $80 \mu \mathrm{g} / \mathrm{ml}$ ) for 24, 48 and $72 \mathrm{~h}$, determined using an MTT assay. In HCT116 cells treated with 2 and $4 \mu \mathrm{g} / \mathrm{ml} \mathrm{ART} \mathrm{for} 48 \mathrm{~h}$, (C) morphological changes were examined using phase-contrast microscope (magnification, x200), (D) DAPI staining and confocal microscopy were used to assess changes in the nucleus, (E) apoptosis was analyzed using flow cytometry, and (F) apoptosis-associated proteins (including caspase 3, PARP, Bax, Bcl-2, Bcl-xL and caspase 9) were examined using western blotting. "P<0.01 vs. corresponding inhibition rate at $24 \mathrm{~h} ;{ }^{~} \mathrm{P}<0.05 \mathrm{vs}$. inhibition rates of 2.5 and $10 \mu \mathrm{g} / \mathrm{ml} \mathrm{ART}$ at the same time point. ART, artesunate; PARP, poly-ADP ribose polymerase; Bcl-2, B-cell lymphoma-2; Bax, Bcl-2 associated X protein; Bcl-xL, Bcl-extra-large protein; 5-Fu, 5-fluorouracil.

ART promotes apoptosis in HCT116 cells via the mitochondrial pathway. Treatment of HCT116 cells with $2,4 \mu \mathrm{g} / \mathrm{ml}$ ART and $0.3 \mu \mathrm{g} / \mathrm{ml} 5$-Fu for $48 \mathrm{~h}$ evidently reduced the number of viable cells. The cell morphology was markedly changed, with cell elongation and membrane foaming observed (Fig. 1C). DAPI staining also revealed the typical apoptotic morphological changes of nuclear condensation, fragmentation, and chromatin shrinkage (Fig. 1D). 
Table I. Growth inhibition rate of HCT116 cells at different ART concentrations and incubation times.

\begin{tabular}{lccc}
\hline & \multicolumn{3}{c}{ Cell growth inhibition rate $(\%)$} \\
\cline { 2 - 4 } ART concentration $(\mu \mathrm{g} / \mathrm{ml})$ & $24 \mathrm{~h}$ & $48 \mathrm{~h}$ & $72 \mathrm{~h}$ \\
\hline 2.5 & $22.95 \pm 6.13$ & $50.40 \pm 2.87^{\mathrm{a}}$ & $57.81 \pm 1.79^{\mathrm{a}}$ \\
10 & $25.38 \pm 5.59$ & $51.56 \pm 6.03^{\mathrm{a}}$ & $63.38 \pm 2.07^{\mathrm{a}}$ \\
40 & $27.07 \pm 1.34$ & $63.60 \pm 7.19^{\mathrm{a}, \mathrm{b}}$ & $79.91 \pm 1.60^{\mathrm{a}, \mathrm{b}}$ \\
80 & $28.30 \pm 4.17$ & $82.24 \pm 5.68^{\mathrm{a}, \mathrm{b}}$ & $90.29 \pm 3.30^{\mathrm{a}, \mathrm{b}}$ \\
\hline
\end{tabular}

${ }^{\mathrm{a}} \mathrm{P}<0.01 \mathrm{vs}$. corresponding inhibition rate at $24 \mathrm{~h}$; ${ }^{\mathrm{b}} \mathrm{P}<0.05 \mathrm{vs}$. inhibition rates of 2.5 and $10 \mu \mathrm{g} / \mathrm{ml}$ ART at the same time point. ART, artesunate.
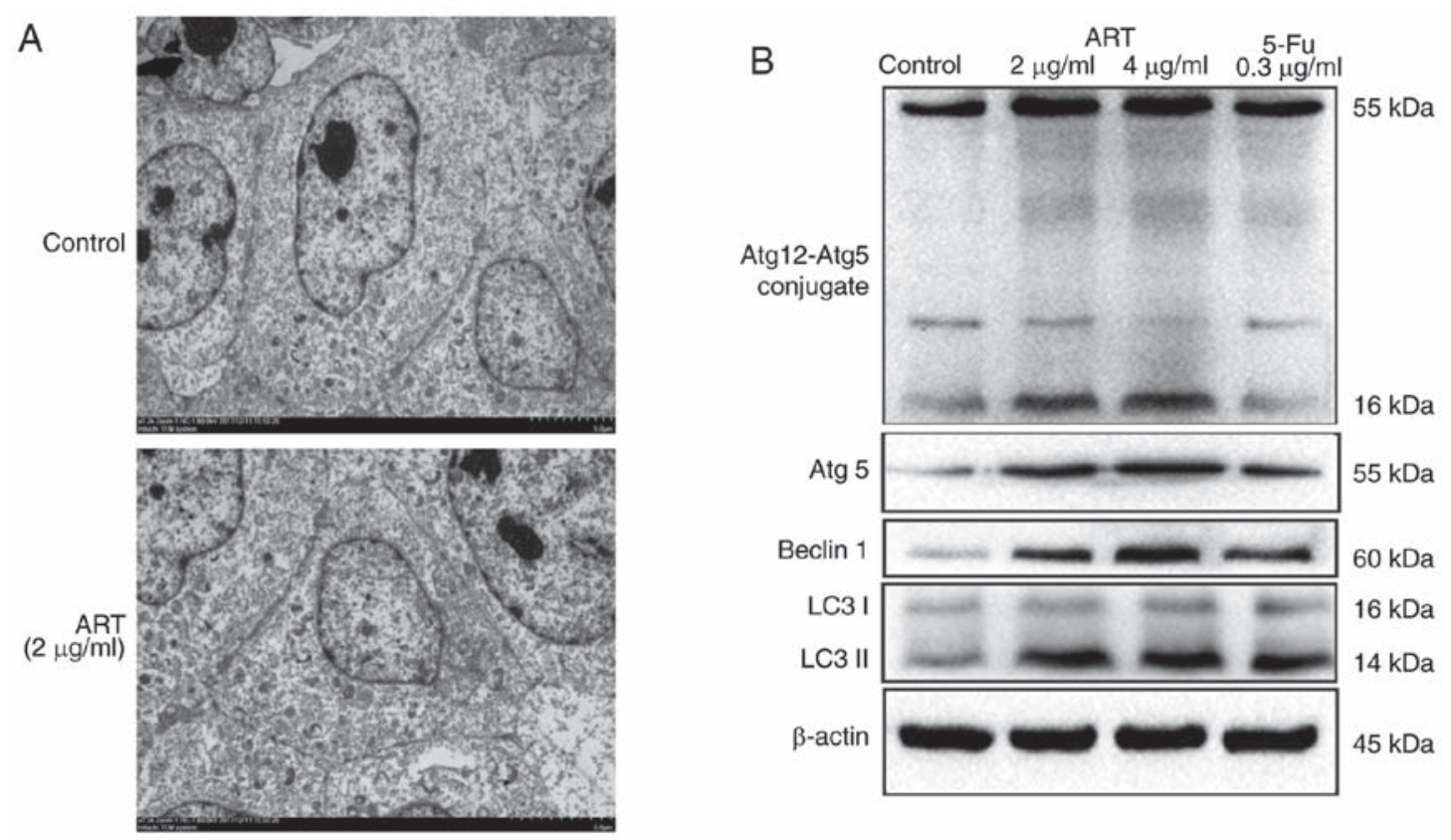

Figure 2. ART promotes autophagy in HCT116 cells. (A) Transmission electron microscopy images displayed autophagosomes in cells treated with $2 \mu \mathrm{g} / \mathrm{ml}$ ART for $48 \mathrm{~h}$. (B) Western blot analysis of cells treated with 2 and $4 \mu \mathrm{g} / \mathrm{ml}$ ART for $48 \mathrm{~h}$ was conducted to determine the LC3, beclin-1, Atg5 and Atg12-Atg5 conjugate expression levels. ART, artesunate; LC3, light chain 3; Atg, autophagy protein; 5-Fu, 5-fluorouracil.

To further confirm the apoptosis-inducing activity of ART, HCT116 cells were subjected to Annexin V-FITC/PI staining with flow cytometry, while 5-Fu was used as the positive proapoptotic drug. The analysis of the results revealed that apoptotic cells accounted for 22.7 and $33.8 \%$ of the cells in early apoptosis (lower right quadrant) following a 48-h treatment with 2 and $4 \mu \mathrm{g} / \mathrm{ml}$ ART, while that accounted for $40.5 \%$ treated with 5-Fu, respectively (Fig. 1E).

To explore the pattern of ART-induced apoptosis of HCT116 cells, the levels of key proteins involved in mitochondria-associated apoptosis were measured using western blotting. As shown in Fig. 1F, procaspase 3 was cleaved to active caspase 3 following ART treatment. In addition, ART increased the proteolytic cleavage of $116 \mathrm{kDa}$ PARP to its $89 \mathrm{kDa}$ fragment, which is a hallmark of apoptosis. ART also increased the protein expression of Bax and decreased the levels of Bcl-2 and Bcl-xL, which are mitochondria-associated proteins. The expression of relative proteins was also increased in the 5-Fu group. These findings suggest that ART activates mitochondria-mediated apoptosis in HCT116 cells.
ART induces autophagy in HCT116 cells. Treatment of HCT116 cells with 2 and $4 \mu \mathrm{g} / \mathrm{ml}$ ART for $48 \mathrm{~h}$ induced the appearance of autophagosomes in the cells (Fig. 2A). The protein expression levels of the autophagy-specific proteins beclin-1 and LC3-I/II, the level of Atg5 and the complexation of Atg12-Atg5 increased following ART exposure, all the protein in 5-Fu group were not markedly increased (Fig. 2B). These findings suggest that ART induced autophagy in HCT116 cells.

Autophagy inhibition promotes ART-induced apoptosis in HCT116 cells. HCT116 cells were treated with $4 \mu \mathrm{g} / \mathrm{ml}$ ART, $4 \mu \mathrm{g} / \mathrm{ml}$ ART $+10 \mu \mathrm{M}$ HCQ (which is a classic autophagy inhibitor) (22), or $10 \mu \mathrm{M}$ HCQ. As shown in Fig. 3A, the inhibition of autophagy enhanced the ART-induced inhibition of cell proliferation $(\mathrm{P}<0.05)$. Furthermore, the examination of HCT116 cells in each group using Annexin V-FITC/PI staining with flow cytometry revealed that the early apoptotic cells accounted for $13.5 \%$ in ART group, but increased to $21.9 \%$ in the ART + HCQ group (Fig. 3B). Examination of 

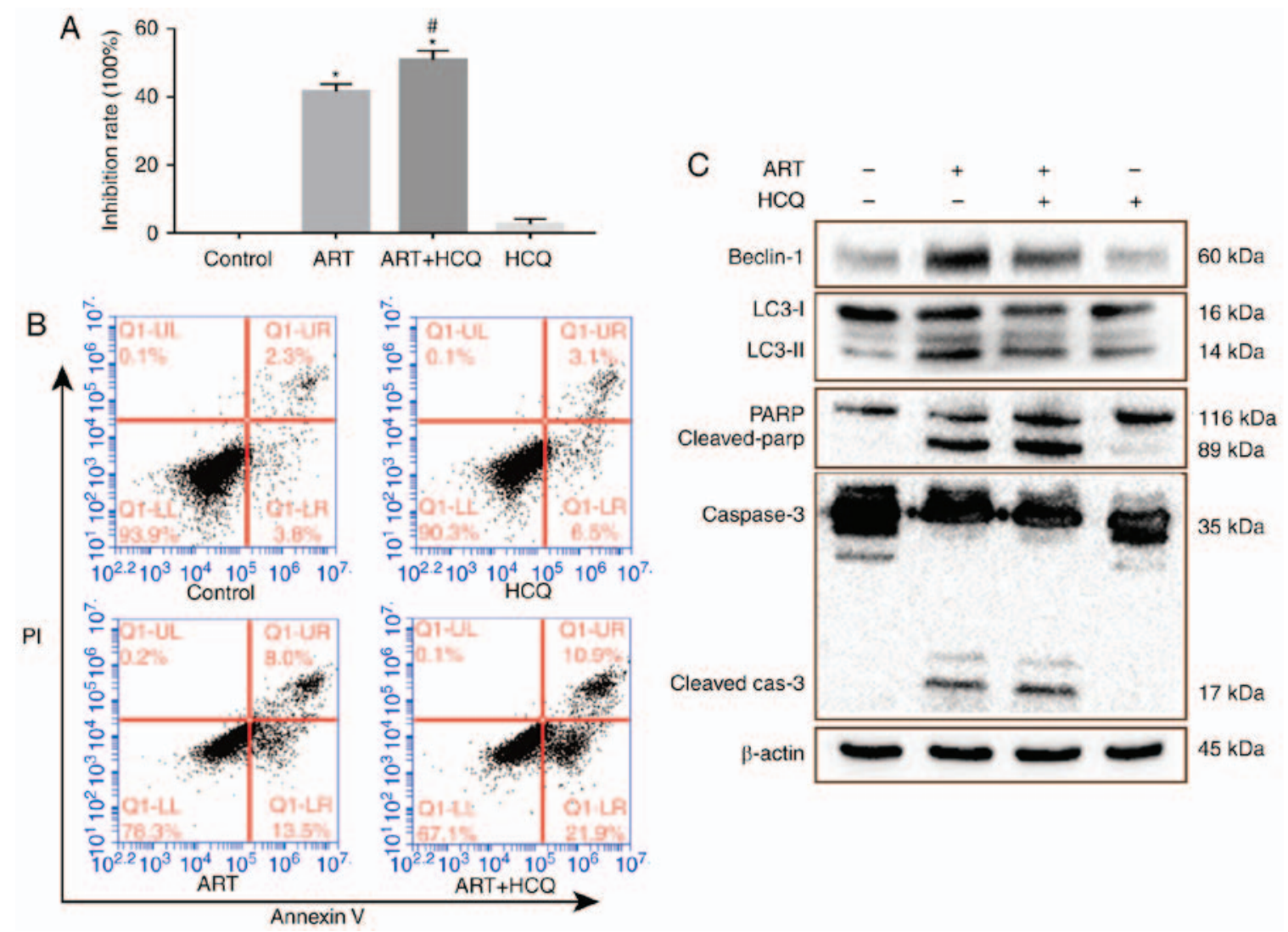

Figure 3. Autophagy inhibition enhances the ART-induced apoptosis of HCT116 cells. (A) MTT assay of cell viability of HCT116 cells pretreated with an autophagy inhibitor (10 $\mu \mathrm{M} \mathrm{HCQ} ; 1 \mathrm{~h})$ followed by ART $(4 \mu \mathrm{g} / \mathrm{ml} ; 48 \mathrm{~h})$. (B) Apoptosis of cells treated with ART $(4 \mu \mathrm{g} / \mathrm{ml})$, ART $(4 \mu \mathrm{g} / \mathrm{ml})+\mathrm{HCQ}(10 \mu \mathrm{M})$, and HCQ $(10 \mu \mathrm{M})$ alone for $48 \mathrm{~h}$ was analyzed by flow cytometry. (C) Cells were incubated with ART, ART+HCQ and HCQ for $48 \mathrm{~h}$, and then caspase 3, PARP, LC3 and beclin-1 protein expression levels were determined using western blotting. ART, artesunate; HCQ, hydroxychloroquine sulfate; PARP, poly-ADP ribose polymerase; LC3, light chain 3.

the expression levels of autophagy- and apoptosis-associated proteins revealed that cleaved caspase 3 and pro-PARP levels increased, while those of LC3-I/II and beclin-1 decreased in the ART+HCQ group (Fig. 3C). These results indicated that the number of apoptotic cells following ART treatment increased when autophagy was inhibited by HCQ.

ART inhibits transplanted tumor growth in mice. The mental state of the animals, intake of food and water, urination, defecation and other activities were not significantly different between the NS, 5-Fu and ART groups. Compared with the control group, the weight of mice in the two treatment groups was not significantly different $(\mathrm{P}>0.05$; data not shown). After a 14-day treatment with the test substances, the mean tumor volumes of the mice in the ART and 5-Fu groups were smaller compared with those in the control group (Fig. 4A and B). At the end of the experiment, the ART and 5-Fu groups exhibited more significant inhibition of tumor growth in comparison with that observed in the control group $(\mathrm{P}<0.01$; Fig. $4 \mathrm{~A}, \mathrm{C}$ and D)

ART promotes the apoptosis of HCT116 cells in transplanted mouse tumors. To elucidate the mechanism of the ART-induced tumor size and weight suppression, a TUNEL assay was performed to examine apoptosis and associated protein expression levels in tumor tissues. ART and 5-Fu promoted the apoptosis of the tumor cells of transplanted mice as evidenced by the detection of TUNEL-positive cells with increased DNA fragmentation in the ART and 5-Fu treated group compared with that in the NS group (Fig. 5A). The immunohistochemical and western blot analyses also revealed that the protein expression levels of cleaved caspase 3 and Bax increased, while the level of Bcl-2 decreased in the ART and 5-Fu group (Fig. 5B and C). The results further confirmed that the antitumor activity of ART in transplanted tumors was associated with increased apoptosis.

\section{Discussion}

The US Food and Drug Administration-approved ART is currently the most potent antimalarial agent available (24). The selective killing of cancer but not normal cells may also be a major advantage of ART, which is already known to be a generally safe and well-tolerated drug, even in the fetus during the first trimester of pregnancy (25). To fully understand the mechanisms of the antitumor actions of ART, the present study investigated for the first time the association between apoptosis and autophagy in HCT-116 cells treated with ART.

In the present study, it was observed that ART significantly inhibited the proliferation of HCT116 cells after $48 \mathrm{~h}$ of treatment, whereas the effect was not significant after $24 \mathrm{~h}$. This result was not consistent with the observations of a previous study, indicating ART significantly inhibited the cell viability of HCT116 after $24 \mathrm{~h}$ of treatment (18), 

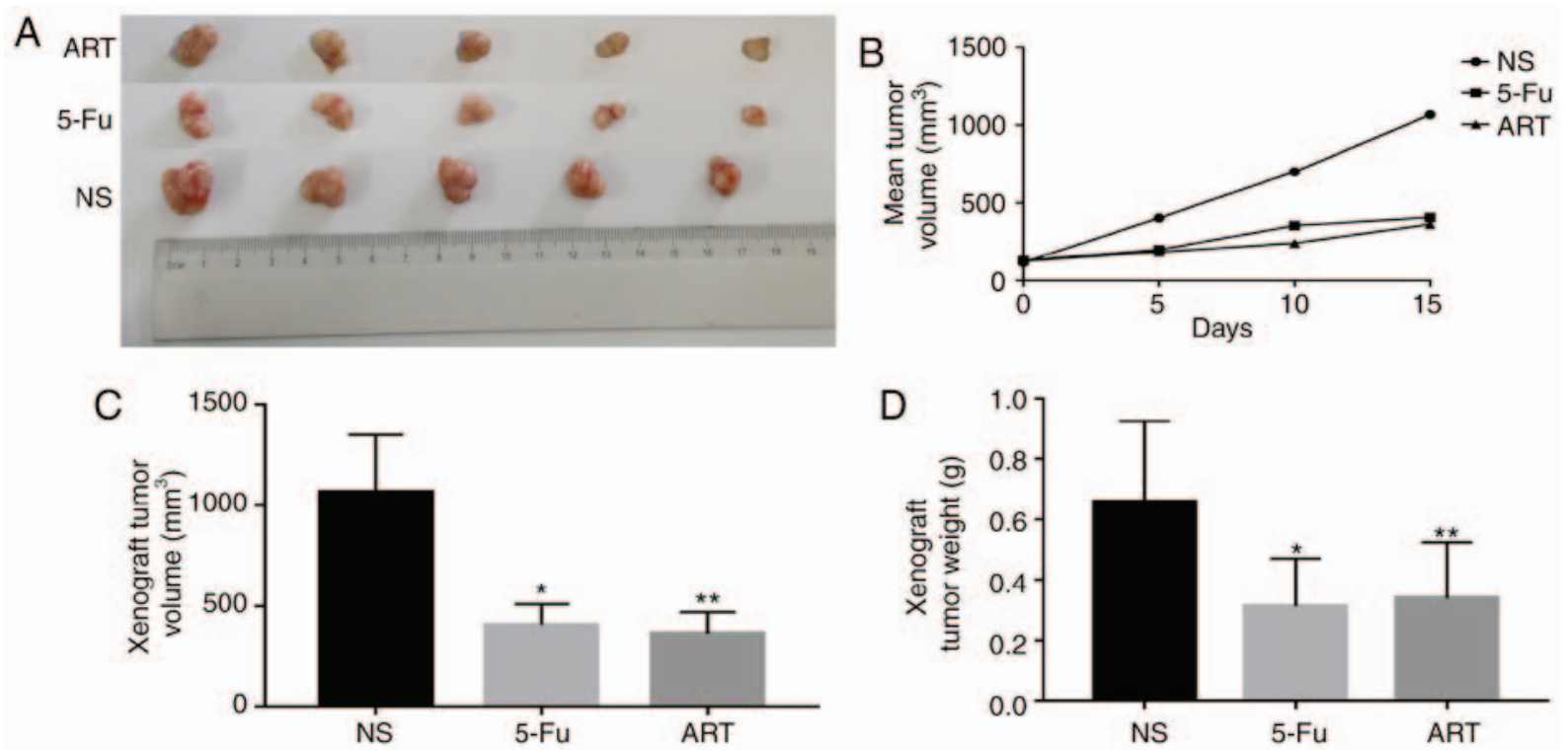

Figure 4. ART inhibits the growth of transplanted HCT116 cell-induced tumors. (A) Images of transplanted tumor in the three groups of mice. (B) Volume change curve of transplanted tumor with increasing treatment time. (C) Volume of transplanted tumors in the three groups at the end of treatment. (D) Weight of transplanted tumor in the three groups at the end of treatment. ${ }^{*} \mathrm{P}<0.01$ vs. NS group; ${ }^{* *} \mathrm{P}<0.01$ vs. NS group.

and a possible explanation for this apparent discrepancy may be the different drug sources. In the current study, a commercially available injectable formulation was used that may contain a number of water-soluble ingredients, while ART as a monomer was used in the previous investigation that was purchased from Sigma-Aldrich (Merck, Darmstadt, Germany) (18).

Apoptosis is an active process of programmed cell death that occurs in multicellular organisms, while the mitochondrial pathway is an important form of apoptosis. Bax and $\mathrm{Bcl}-2$ proteins are members of the Bcl-2 family, which consists of pro- and anti-apoptotic proteins that exert opposing effects on the mitochondria (26). Upregulation of Bax and caspase 3, and downregulation of Bcl-2 may result in cell apoptosis (27). Bax permeabilizes the mitochondrial outer membrane, releasing pro-apoptotic factors that activate caspases (28), which can then active the nuclear protein PARP (29). In the present study, HCT116 cell apoptosis following intervention with ART was assessed by DAPI staining and flow cytometry in vitro. The western blot analysis revealed that the protein expression levels of Bax, cleaved caspase 3 and cleaved PARP were increased, while those of Bcl-2 and Bcl-xL were decreased, which are all mitochondria-associated proteins. In vivo, ART significantly inhibited tumor size and weight, and evidently induced the apoptosis of transplanted tumor cells by increasing the levels of proteins associated with the mitochondrial pathway. Therefore, the present study suggested that ART induces the apoptosis of HCT116 cells through the mitochondrial pathway, which was also observed in other cells, including HepG2 (19), Eca109 and Ec9706 cells (17).

Autophagy is important in normal development and cellular response to environmental stimuli. It is an intracellular degradation system that delivers cytoplasmic constituents to the lysosome and involves the formation of a double-membrane vesicle, also termed autophagosome. Autophagosomes encapsulate the cytoplasm and organelles, and fuse with lysosomes, thereby degrading the vesicle contents (30). This physiological process is mediated by the interaction of various molecules, such as beclin1 and LC3, as well as Atg5 and $\operatorname{Atg} 12$ (21), which form a conjugate that is essential for autophagosome formation (31). In CRC cells with high microsatellite instability (such as HCT116 cells), Atg5 and Atg12 mutations may contribute to the tumor progression by deregulating the autophagy process (32). In the present study, intracellular autophagosomes were identified using TEM, indicating that autophagy occurred in the ART-treated HCT116 cells. In addition, western blotting revealed that the levels of LC3-II, beclin-1 and Atg5, as well as the Atg5-Atg12 conjugate, increased after HCT116 cells were treated with ART. These results indicate that ART induced autophagy in HCT116 cells, which was also previously reported in breast cancer cells (13).

Autophagy and apoptosis are well-regulated biological processes that have important roles in tumor development and progression. Whether autophagy is pro-tumorigenic or antitumorigenic in cancer development and therapy remains unclear. Several studies have demonstrated that autophagy has an anti-apoptotic role in numerous types of cancer (33-36), whereas other studies reported its essential pro-apoptotic role in certain cancer tissues $(22-23,37,38)$. In the current study, it was observed that the inhibition of autophagy enhanced the ART-induced inhibition of cell proliferation. In addition, the percentage of early apoptotic cells increased after co-treatment with ART and HCQ compared with that of cells treated with ART alone. Similarly, autophagy and apoptosis are closely linked in the expression of specific proteins. The inhibition of beclin-1 by RNAi suppressed autophagic activity and proliferation, but promoted apoptosis in the CRC HCT116 and SW620 cell lines (39). The present study revealed that the protein expression of cleaved caspase 3 and PARP increased, while that of beclin1 and LC3 decreased in the ART + HCQ group compared with cells treated with ART alone. Therefore, 

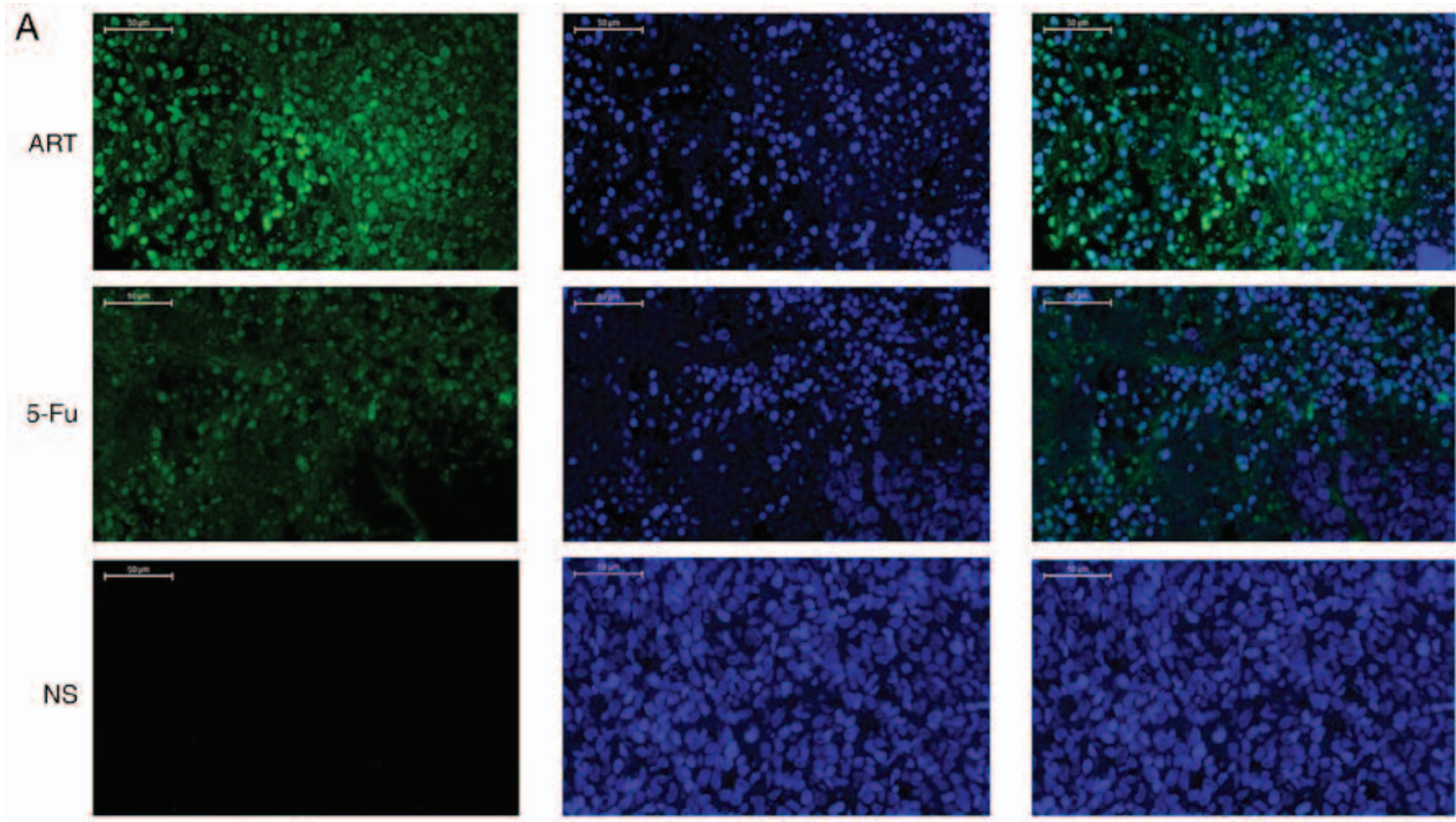

DAPI

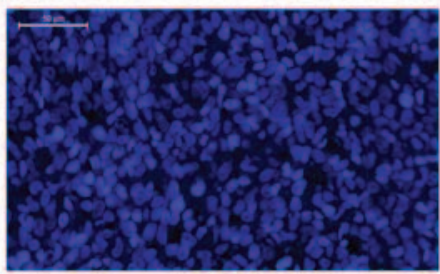

Tunel
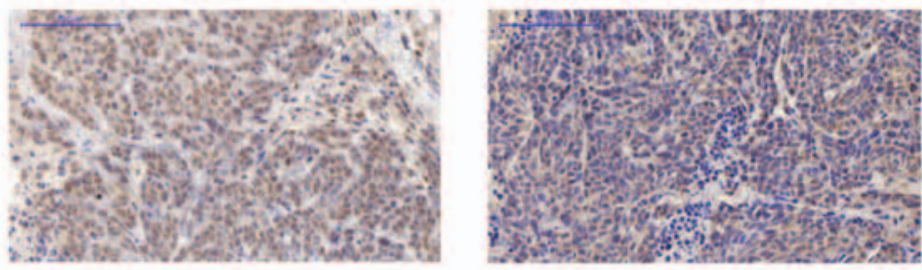

B
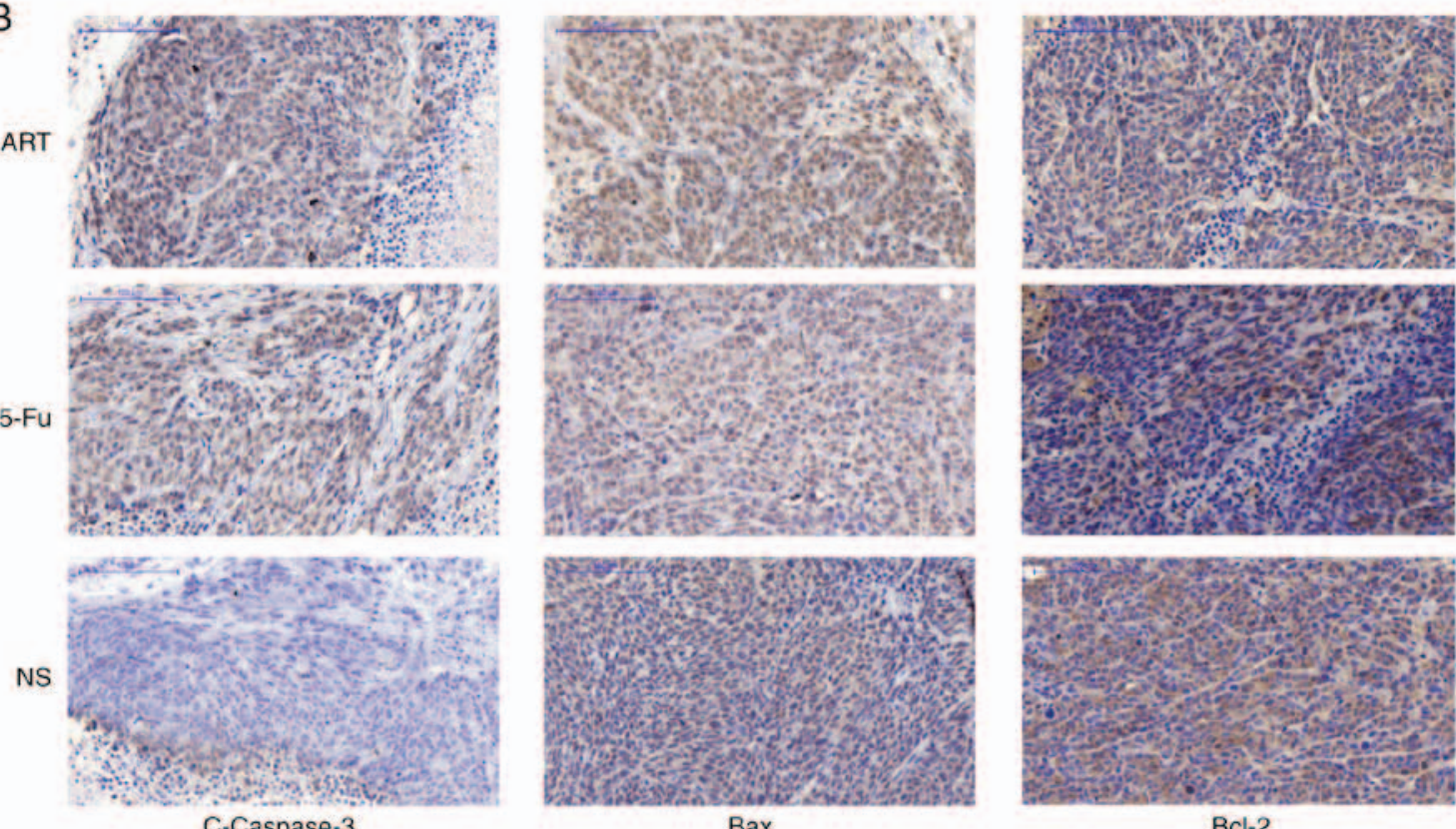

C

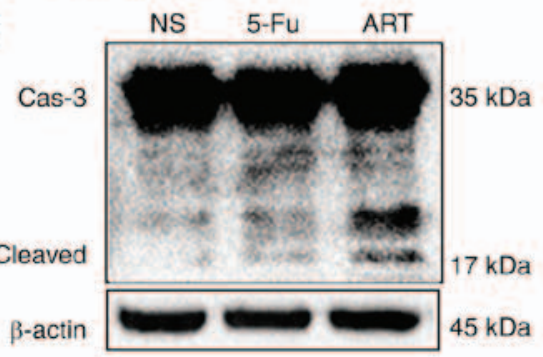

Bax

$\mathrm{BCl}-2$

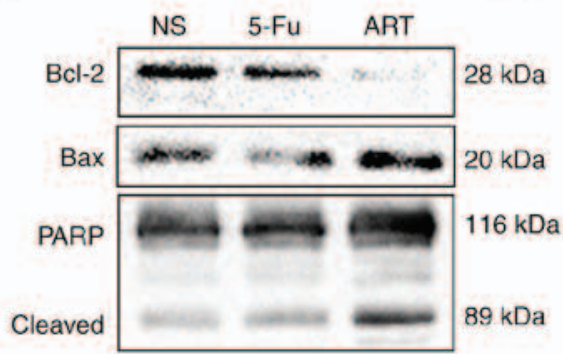

Figure 5. ART induces apoptosis in HCT116 cell-induced tumors in mice. (A) Apoptosis was determined using TUNEL assay in the three groups. Green fluorescence indicates TUNEL-positive cells in the microscopic fields (magnification, x400). (B) Apoptosis-associated protein expression was determined using immunohistochemistry. (C) Western blotting was used to examine the protein expression of cleaved caspase 3, PARP, Bax and Bcl-2. ART, artesunate; PARP, poly-ADP ribose polymerase; Bcl-2, B-cell lymphoma-2; Bax, Bcl-2 associated X protein; 5-Fu, 5-fluorouracil.

the results are consistent with those of other published studies, demonstrating that autophagy inhibition promotes the apoptosis of certain cancer cells (33-36).
In conclusion, ART induced apoptosis both in vitro and in vivo, as well as autophagy in HCT116 cells. However, autophagy protected HCT116 cells from the apoptosis induced 
by ART. These findings provide a basis for further investigation and development of ART as a potential drug for CRC. Furthermore, co-administration of ART with an autophagy inhibitor may improve the efficacy of ART; however, further research would be required to verify the antitumor activity of ART.

\section{Acknowledgements}

Not applicable.

\section{Funding}

This study was supported by the National Natural Science Foundation of China (grant no. 81373645, 81573978). This study was also supported by the Priority Academic Program Development of Jiangsu Higher Education Institutions and Jiangsu Province Special Program of Medical Science (grant no. BL2014100) and by the Peak Academic Talents plan (grant no. BRA2017536) of the Jiangsu Province Hospital of TCM (Jiangsu, China).

\section{Availability of data and materials}

All data generated or analyzed during this study are included in this published article.

\section{Authors' contributions}

FJ and YC were responsible for conception and design of the experiments. FJ and JZ performed the experiments. FJ and DZ analyzed the data. DZ and ML supplied reagents, materials and analysis tools. FJ and YC wrote this manuscript. FJ and ML edited all the pictures. JZ and DZ revised the manuscript critically. All authors have agreed to the publication of this manuscript.

\section{Ethics approval and consent to participate}

The experimental animal protocols were reviewed and approved by the Animal Ethics Committee of the Affiliated Hospital of Nanjing University of TCM (Jiangsu, China; 2017-DW-11).

\section{Patient consent for publication}

Not applicable.

\section{Competing interests}

The authors declare no conflict of interests regarding the publication of this manuscript.

\section{References}

1. Torre LA, Bray F, Siegel RL, Ferlay J, Lortet-Tieulent J and Jemal A: Global cancer statistics, 2012. CA Cancer J Clin 65: 87-108, 2015.

2. Coghlin C and Murray GI: Biomarkers of colorectal cancer: Recent advances and future challenges. Proteomics Clin Appl 9: 64-71, 2015.
3. Efferth $\mathrm{T}$ and Kaina B: Toxicity of the antimalarial artemisinin and its dervatives. Crit Rev Toxicol 40: 405-421, 2010.

4. Efferth T, Rücker G, Falkenberg M, Manns D, Olbrich A, Fabry U and Osieka R: Detection of apoptosis in KG-1a leukemic cells treated with investigational drugs. Arzneimittelforschung 46: 196-200, 1996.

5. Xu N, Zhou X, Wang S, Xu LL, Zhou HS and Liu XL: Artesunate induces SKM-1 cells apoptosis by inhibiting hyperactive $\beta$-catenin signaling pathway. Int J Med Sci 12: 524-529, 2015.

6. Wang Y, Yang J, Chen L, Wang J, Wang Y, Luo J, Pan L and Zhang $\mathrm{X}$ : Artesunate induces apoptosis through caspase-dependent and -independent mitochondrial pathways in human myelodysplastic syndrome SKM-1 cells. Chem Biol Interact 219: 28-36, 2014.

7. Papanikolaou X, Johnson S, Garg T, Tian E, Tytarenko R, Zhang Q, Stein C, Barlogie B, Epstein J and Heuck C: Artesunate overcomes drug resistance in multiple myeloma by inducing mitochondrial stress and non-caspase apoptosis. Oncotarget 5: 4118-4128, 2014

8. Kim C, Lee JH, Kim SH, Sethi G and Ahn KS: Artesunate suppresses tumor growth and induces apoptosis through the modulation of multiple oncogenic cascades in a chronic myeloid leukemia xenograft mouse model. Oncotarget 6: 4020-4035, 2014.

9. Wang ZC, Liu Y, Wang H, Han QK and Lu C: Research on the relationship between artesunate and Raji cell autophagy and apoptosis of Burkitt's lymphoma and its mechanism. Eur Rev Med Pharmacol Sci 21: 2238-2243, 2017.

10. Zuo W, Wang Z-Z and Xue J: Artesunate induces apoptosis of bladder cancer cells by miR-16 regulation of COX-2 expression. Int J Mol Sci 15: 14298-14312, 2014.

11. Jeong DE, Song HJ, Lim S, Lee SJ, Lim JE, Nam DH, Joo KM, Jeong BC, Jeon SS, Choi HY and Lee HW: Repurposing the anti-malarial drug artesunate as a novel therapeutic agent for metastatic renal cell carcinoma due to its attenuation of tumor growth, metastasis, and angiogenesis. Oncotarget 6: 33046-33064, 2015.

12. Zhang LX, Liu ZN, Ye J, Sha M, Qian H, Bu XH, Luan ZY, $\mathrm{Xu} \mathrm{XL}$, Huang $\mathrm{AH}$, Yuan DL, et al: Artesunate exerts an anti-immunosuppressive effect on cervical cancer by inhibiting PGE2 production and Foxp3 expression. Cell Biol Int 38: 639-646, 2014.

13. Chen K, Shou L-M, Lin F, Duan WM, Wu MY, Xie X, Xie YF, Li W and Tao M: Artesunate induces G2/M cell cycle arrest through autophagy induction in breast cancer cells. Anticancer Drugs 25: 652-662, 2014.

14. Wang Z, Wang C, Wu Z, Xue J, Shen B, Zuo W, Wang Z and Wang SL: Artesunate suppresses the growth of prostatic cancer cells through inhibiting androgen receptor. Biol Pharm Bull 40: 479-485, 2017.

15. Roh JL, Kim EH, Jang H and Shin D: Nrf2 inhibition reverses the resistance of cisplatin-resistant head and neck cancer cells to artesunate-induced ferroptosis. Redox Biol 11: 254-262, 2017.

16. Pang Y, Qin G, Wu L, Wang X and Chen T: Artesunate induces ROS-dependent apoptosis via a Bax-mediated intrinsic pathway in Huh-7 and Hep3B cells. Exp Cell Res 347: 251-260, 2016.

17. Liu L, Zuo LF, Zuo J and Wang J: Artesunate induces apoptosis and inhibits growth of Eca109 and Ec9706 human esophageal cancer cell lines in vitro and in vivo. Mol Med Rep 12: 1465-1472, 2015

18. Chen X, Wong YK, Lim TK, Lim WH, Lin Q, Wang J and Hua Z: Artesunate activates the intrinsic apoptosis of HCT116 cells through the suppression of fatty acid synthesis and the NF-kappaB pathway. Molecules 22: E1272, 2017.

19. Qin G, Wu L, Liu H, Pang Y, Zhao C, Wu S, Wang X and Chen T: Artesunate induces apoptosis via a ROS-independent and Bax-mediated intrinsic pathway in HepG2 cells. Exp Cell Res 336: 308-317, 2015.

20. O'Donovan TR, Rajendran S, O'Reilly S, O'Sullivan GC and McKenna SL: Lithium modulates autophagy in esophageal and colorectal cancer cells and enhances the efficacy of therapeutic agents in vitro and in vivo. PLoS One 10: e0134676, 2015.

21. Coker-Gürkan A, Arisan ED, Obakan P, Akalın K, Özbey U and Palavan-Unsal N: Purvalanol induces endoplasmic reticulum stress-mediated apoptosis and autophagy in a time-dependent manner in HCT116 colon cancer cells. Oncol Rep 33: 2761-2770, 2015.

22. Ranjan A and Srivastava SK: Penfluridol suppresses pancreatic tumor growth by autophagy-mediated apoptosis. Sci Rep 6: 26165, 2016. 
23. Wang Y, Luo Q, He X, Wei H, Wang T, Shao J and Jiang X: Emodin induces apoptosis of colon cancer cells via induction of autophagy in a ROS-dependent manner. Oncol Res, 2017 Jul 25. Doi: $10.3727 / 096504017 X 15009419625178$.

24. Rosenthal PJ: Artesunate for the treatment of severe falciparum malaria. N Engl J Med 358: 1829-1836, 2008.

25. Clark RL: Embryotoxicity of the artemisinin antimalarials and potential consequences for use in women in the first trimester. Reprod Toxicol 28: 285-296, 2009.

26. Oh KJ, Barbuto S, Pitter K, Morash J, Walensky LD and Korsmeyer SJ: A membrane-targeted BID BCL-2 homology 3 peptide is sufficient for high potency activation of BAX in vitro. J Biol Chem 281: 36999-37008, 2006.

27. Siddiqui WA, Ahad A and Ahsan H: The mystery of BCL2 family: Bcl-2 proteins and apoptosis: An update. Arch Toxicol 89: 289-317, 2015.

28. Martinou JC and Green DR: Breaking the mitochondrial barrier. Nat Rev Mol Cell Biol 2: 63-67, 2001.

29. Chaitanya GV, Steven AJ and Babu PP: PARP-1 cleavage fragments: Signatures of cell-death proteases in neurodegeneration. Cell Commun Signal 8: 31, 2010.

30. Mizushima N: Autophagy: Process and function. Genes Dev 21: 2861-2873, 2007

31. Hanada T and Ohsumi Y: Structure-function relationship of Atg12, a ubiquitin-like modifier essential for autophagy. Autophagy 1: 110-118, 2005.

32. Kang MR, Kim MS, Oh JE, Kim YR, Song SY, Kim SS, Ahn CH, Yoo NJ and Lee SH: Frameshift mutations of autophagy-related genes ATG2B, ATG5, ATG9B and ATG12 in gastric and colorectal cancers with microsatellite instability. J Pathol 217: 702-706, 2009.

33. Zhang Y, Chen P, Hong H, Wang L, Zhou Y and Lang Y: JNK pathway mediates curcumin-induced apoptosis and autophagy in osteosarcoma MG63 cells. Exp Ther Med 14: 593-599, 2017.
34. Jung HJ, Kang JH, Choi S, Son YK, Lee KR, Seong JK, Kim SY and Oh SH: Pharbitis Nil (PN) induces apoptosis and autophagy in lung cancer cells and autophagy inhibition enhances PN-induced apoptosis. J Ethnopharmacol 208: 253-263, 2017.

35. Hseu YC, Tsai TJ, Korivi M, Liu JY, Chen HJ, Lin CM, Shen YC and Yang HL: Antitumor properties of Coenzyme Q0 against human ovarian carcinoma cells via induction of ROS-mediated apoptosis and cytoprotective autophagy. Sci Rep 7: 8062, 2017.

36. Cave DD, Desiderio V, Mosca L, Ilisso CP, Mele L, Caraglia M, Cacciapuoti G and Porcelli M: S-Adenosylmethionine-mediated apoptosis is potentiated by autophagy inhibition induced by chloroquine in human breast cancer cells. J Cell Physiol 233: 1370-1383, 2017.

37. Huang CC, Lee CC, Lin HH, Chen MC, Lin CC and Chang JY: Autophagy-regulated ROS from xanthine oxidase acts as an early effector for triggering late mitochondria-dependent apoptosis in cathepsin S-targeted tumor cells. PLoS One 10: e0128045, 2015.

38. Won SJ, Yen CH, Liu HS, Wu SY, Lan SH, Jiang-Shieh YF, Lin CN and Su CL: Justicidin A-induced autophagy flux enhances apoptosis of human colorectal cancer cells via class III PI3K and Atg5 pathway. J Cell Physiol 230: 930-946, 2015.

39. Liu L, Zhao WM, Yang XH, Sun ZQ, Jin HZ, Lei C, Jin B and Wang HJ: Effect of inhibiting Beclin-1 expression on autophagy, proliferation and apoptosis in colorectal cancer. Oncol Lett 14: 4319-4324, 2017

(i) $($ ) This work is licensed under a Creative Commons Attribution-NonCommercial-NoDerivatives 4.0 International (CC BY-NC-ND 4.0) License. 